\title{
APPLICATION OF MEMETIC ALGORITHMS IN MULTI-CRITERIA STRUCTURAL OPTIMIZATION
}

The goal of this paper is to present new innovative algorithms for discrete structural optimization of problems involving multi-criteria objective function, which contains stress, displacement and frequency constraints. The algorithm is tested on a shell finite element structure, whose optimization variables are geometrical parameters and shell thicknesses. Performance of memetic algorithm will be compared with genetic algorithm.

Keywords: Memetic algorithm, genetic algorithm, structural optimization, multi-criteria optimization, modal analysis, buckling analysis, static analysis.

\section{Introduction}

Problems solved by engineering practice often incorporate multiple constraints which have to be taken into account [1 - 5]. Problems involving multiple criteria can be often divided into multiple sub-problems, which have to be calculated separately. Multi-criteria objective function has to take into account all subproblems to properly evaluate quality of a solution [6]. Each subproblem affects the character of the objective function and so it can be hard to choose a single optimization method, which would be equally effective for all sub-problems. Modern optimization methods like Memetic Algorithms (MA) can be used to overcome these difficulties. Memetic algorithms are optimization methods which combine global search capabilities of global optimization methods like genetic algorithms or particle swarm optimization and fast converging local search methods like conjugate gradient method or simplex method [7].

\section{Multi-criteria objective function}

The problem solved in this paper is constrained by stress constraint, displacement constraint, modal constraint and buckling constraint. Objective function is penalized when one of the constraints is violated. The stress constraint requires that the maximal stress is lower than stress limit $\sigma_{L}$. Displacement constraint requires that the maximal displacement is lower than displacement limit $u_{L}$. Modal constraint requires that natural frequencies are outside of forbidden ranges, whose lower and upper boundaries are calculated from excitation frequencies by subtracting and adding $10 \%$ of excitation frequencies. Buckling constraint requires that Buckling Load Factor (BLF) is higher than buckling load factor limit $B L F_{L}$.

The objective function is initialized as mass of the frame:

$f(x)=$ mass

If the stress constraint is violated, the objective function is penalized as follows:

$f(x)=f(x)+10 \cdot f(x) \cdot\left(\frac{\left(\sigma_{\max }-\sigma_{L}\right)}{\sigma_{L}}\right)$

If the displacement constraint is violated, the objective function is penalized as follows:

$f(x)=f(x)+10 \cdot f(x) \cdot\left(\frac{\left(u_{\max }-u_{L}\right)}{u_{L}}\right)$

Lower and upper limit is calculated for each excitation frequency. Number of natural frequencies which fall into forbidden ranges is used to penalize the objective function as follows:

$f(x)=f(x) \cdot f(x) \cdot$ violaton $_{\text {count }}$

If the buckling constraint is violated, the objective function is penalized as follows:

$f(x)=f(x)+10 \cdot f(x) \cdot\left(\frac{B L F_{L}}{B L F}\right)$

\footnotetext{
* ${ }^{1}$ Peter Pechac, ${ }^{1}$ Milan Saga, ${ }^{1}$ Peter Weis, ${ }^{2}$ Tomasz Domanski, ${ }^{2}$ Marcin Kubiak

${ }^{1}$ Faculty of Mechanical Engineering, University of Zilina, Slovakia

${ }^{2}$ Faculty of Mechanical Engineering and Computer Science, Czestochowa University of Technology, Poland

E-mail: peter.pechac@fstroj.uniza.sk
} 


\section{Description of the proposed memetic algorithm}

The memetic algorithm used in this paper was based on a genetic algorithm with rank selection, uniform crossover and non-uniform Cauchy mutation [8]. Probability of crossover was calculated based on diversity and the value of objective function. Probability of mutation decreased exponentially with spent computational time and iteration steps, starting at $\mathrm{p}_{\text {mut }}=0.3$. The best $10 \%$ of GA population was copied to the next generation without change, to ensure that the best solutions were not lost. The worst $30 \%$ of GA population was deleted. The remaining $70 \%$ of solutions are used to generate new solutions using crossover and mutation operators. $10 \%$ of the remaining solutions were improved by local search. Additional solutions were randomly generated by White Space Search algorithm (WSS) [9] to complete the original size of GA population. Solutions which were improved by local search (LS) were chosen with probability proportional to their diversity and value of objective function. Local search methods were allowed to improve all optimization variables, however each local search method could run only for 5 iteration steps. Local search methods were chosen automatically with probability proportional to their performance, which was evaluated in real time. The algorithm was using four local search methods: Pattern Search (PS), Nelder-Mead method (NM), DaiYuan version of Nonlinear Conjugate Gradient Method (NCG) with line search [10] and Particle Swarm Optimization (PSO) with inertia parameters tuned for local search [11 - 12]. The search radius of PS, size of simplex for NM, maximal reach of line search for NCG and initial velocity of PSO were linearly decreasing with GA iteration steps [13]. The features of the algorithm were set to promote exploration of optimization space in the beginning and gradually transform to intensive exploitation in the final stage of optimization. The scheme of the algorithm is depicted in Fig. 1.

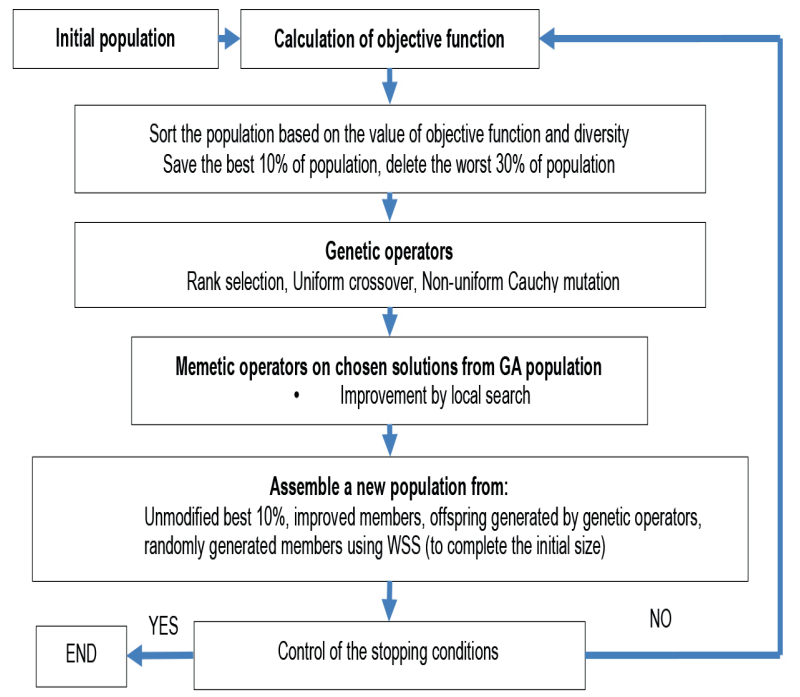

Fig. 1 Scheme of memetic algorithm

\section{Numerical testing}

The proposed algorithm was tested on a frame structure modeled by thin shell finite elements. The steel frame was holding an electric motor and transmission [14]. The original design of structure was made from normalized steel profiles as can be seen in Fig. 2. Weight of the original frame was approximately $2733.5 \mathrm{~kg}$ (calculated from CAD model). The new design was based on welded sheet metal, which is more suitable for optimization and allows greater weight savings.

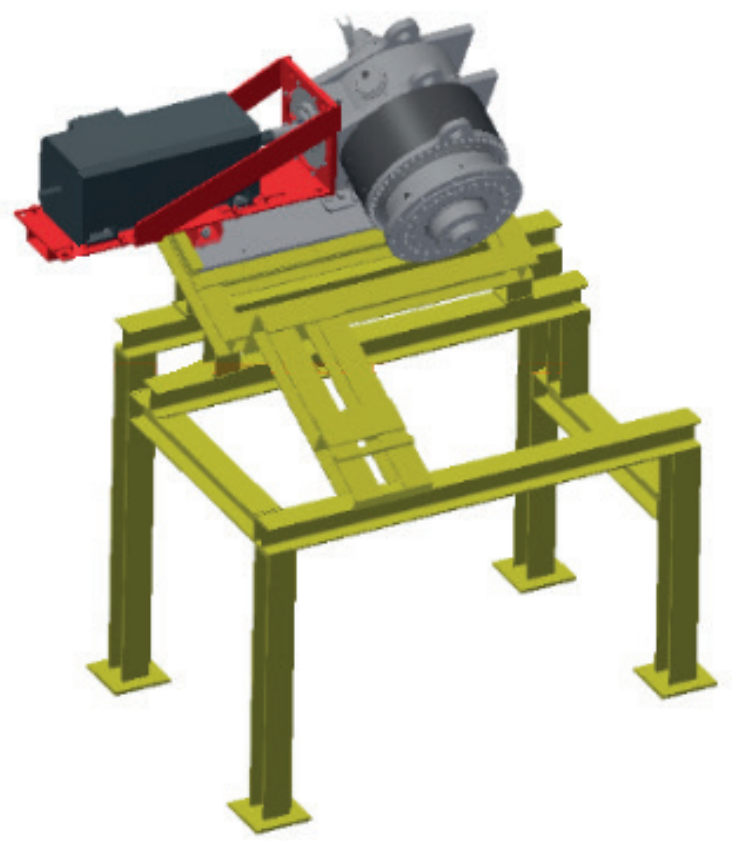

Fig. 2 Original design of frame with electric motor and transmission

The frame was loaded by its own mass, mass of the electromotor, mass of transmission and output moment of 200 $\mathrm{kNm}$. The four pillars were fixed to the ground by constraining all displacement and rotational degrees of freedom. The output frequency of electromotor was $21.16 \mathrm{~Hz}$ and the output frequency of transmission was $0.388 \mathrm{~Hz}$. The electromotor and transmission were modeled by mass point connected to a base plate by linear beam elements. The base plate was modeled by $3 \mathrm{D}$ linear tetrahedron elements and bonded contact was used to connect the base plate to the frame. The frame itself was modeled by linear triangular thin shell elements. Linear elastic material model was used for $3 \mathrm{D}$ model of base plate and shell elements of the frame. Young's modulus of elasticity E $=210000 \mathrm{MPa}$, Poisson's number $\mu=0.3$, density $\rho=7850 \mathrm{~kg} / \mathrm{m}^{3}$. Boundary conditions and optimization variables can be seen in Figs. 3, 4 and 5. 


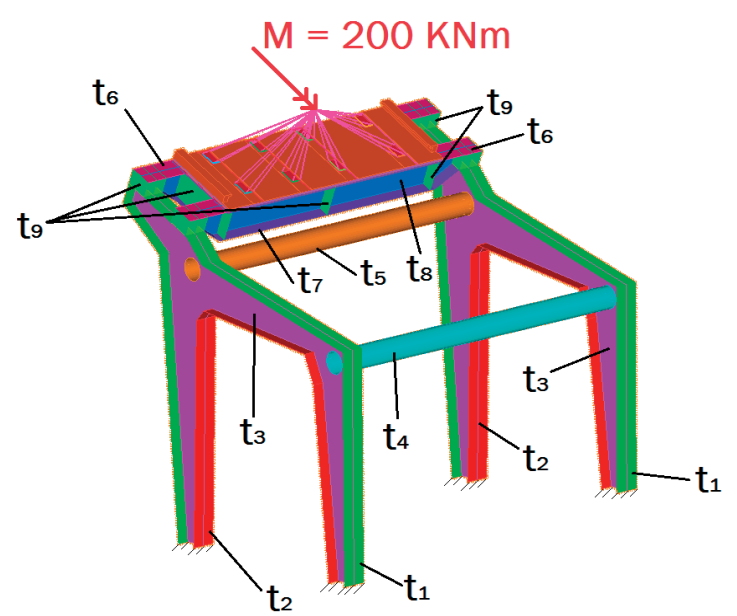

Fig. 3 Thickness optimization variables $\left(t_{l}-t_{\theta}\right)$ and boundary conditions

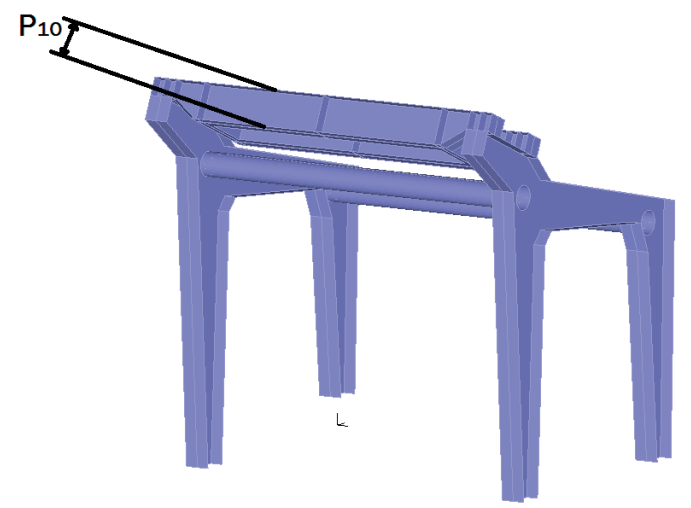

Fig. 4 Geometric optimization variable $P_{10}$

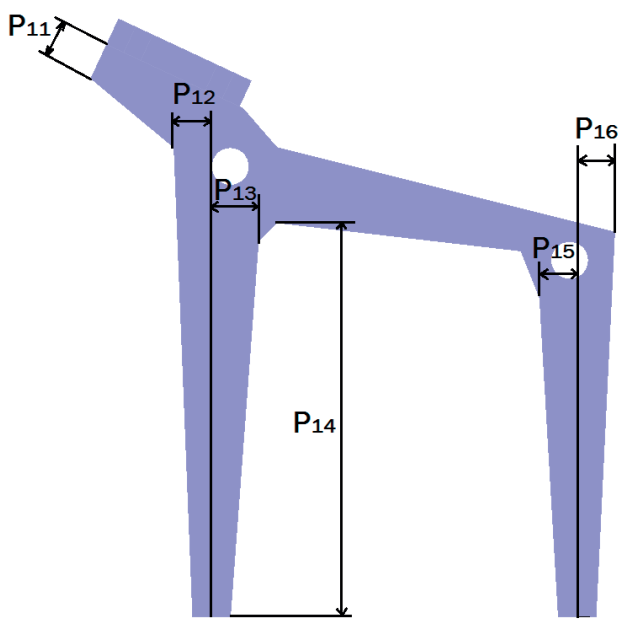

Fig. 5 Geometric optimization variables $P_{11}-P_{16}$

The problem had 16 optimization variables - 9 thickness variables and 7 geometric variables. Shell thicknesses were divided into 26 discrete values from 5 to $30 \mathrm{~mm}$. Geometrical parameters were also divided into discrete values.
The stress limit was set to $\sigma_{\mathrm{L}}=120 \mathrm{MPa}$, displacement limit was $\mathrm{u}_{\mathrm{L}}=5 \mathrm{~mm}$, buckling load factor limit $\mathrm{BLF}_{\mathrm{L}}=6$. Forbidden frequency ranges were set to $\pm 10 \%$ of excitation frequencies of $0.388 \mathrm{~Hz}$ and $21.16 \mathrm{~Hz}$ resulting in forbidden ranges $\mathrm{R}_{1}=<0.3495$, $0.4272>\mathrm{Hz}$ and $\mathrm{R}_{2}=\langle 19.05,23.2833\rangle \mathrm{Hz}$.

All FEM simulations were calculated using commercial FEM software ADINA in parallel using custom developed system in Matlab with use of Matlab Parallel Toolbox. Computational hardware consisted of two high performance server machines with total of $128 \mathrm{CPU}$ cores and $512 \mathrm{~GB}$ RAM. Available license of Matlab Parallel Toolbox allowed to run totally 24 FEM simulations in parallel.

Initial numerical testing was performed using 100 solutions in GA population and maximum computational time was set to 24 hours. The results of initial tests can be seen in following Table 1 and Figs. $6-12$.

Best value of objective function and number of objective function evaluations MA vs. GA

Table 1

\begin{tabular}{|c|c|c|}
\hline Method & Best $\mathbf{f}(\mathbf{x})$ & Number of $\mathbf{f}(\mathbf{x})$ evaluations \\
\hline MA & 1411.2129 & 4275 \\
\hline GA & 1468.378 & 5731 \\
\hline
\end{tabular}

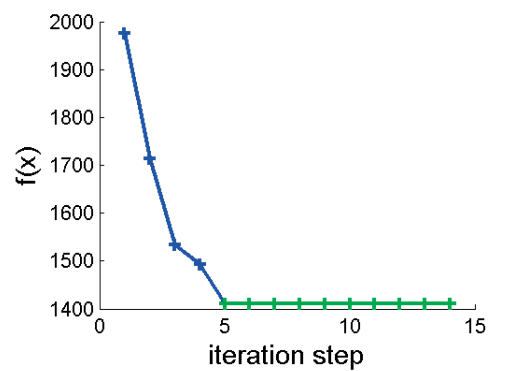

Fig. 6 Initial MA run - History of objective function vs. iteration step
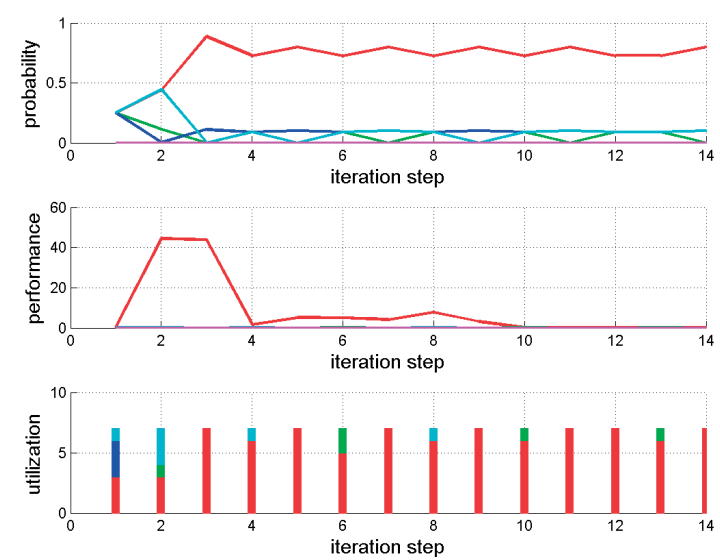

Fig. 7 Initial MA run - History of local search - probability of using $L S$ methods, performance of $L S$ methods and real utilization of $L S$ methods (red - PS, green - NM, blue - NCG, cyan - PSO) 


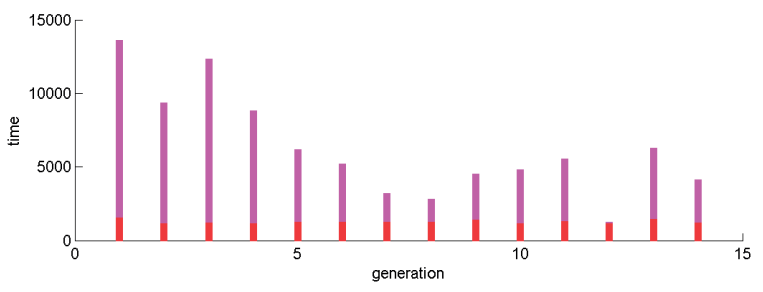

Fig. 8 Initial MA run - History of computational time spent by GA and LS evaluations (red - GA, magenta - LS)

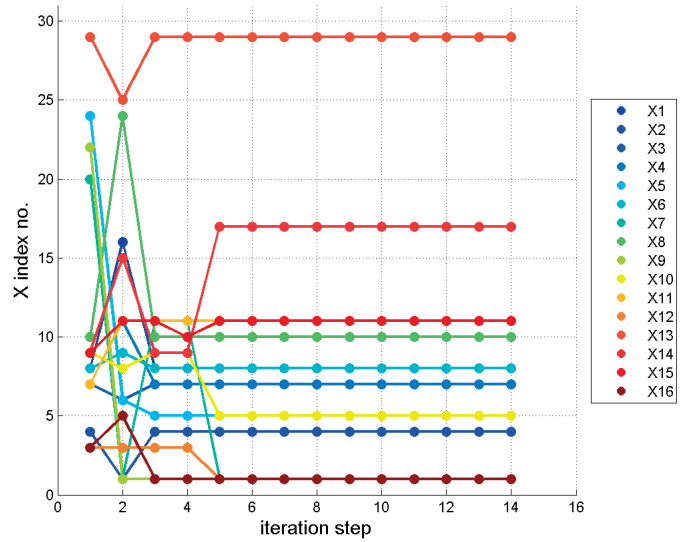

Fig. 9 Initial MA run - History of optimization variables (chosen indexes from vectors of discrete design variables)

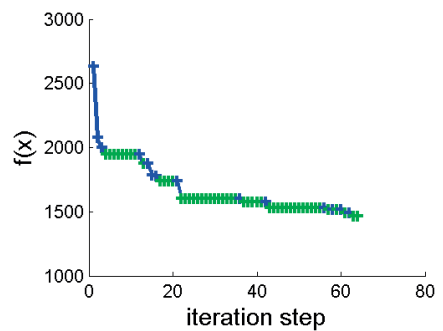

Fig. 10 Initial GA run - History of objective function vs. iteration step

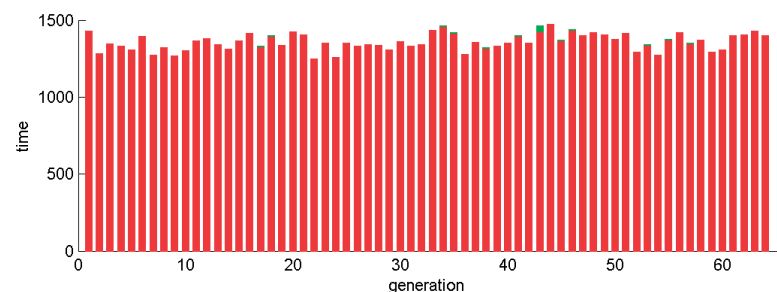

Fig. 11 Initial GA run - History of computational time spent by GA evaluations

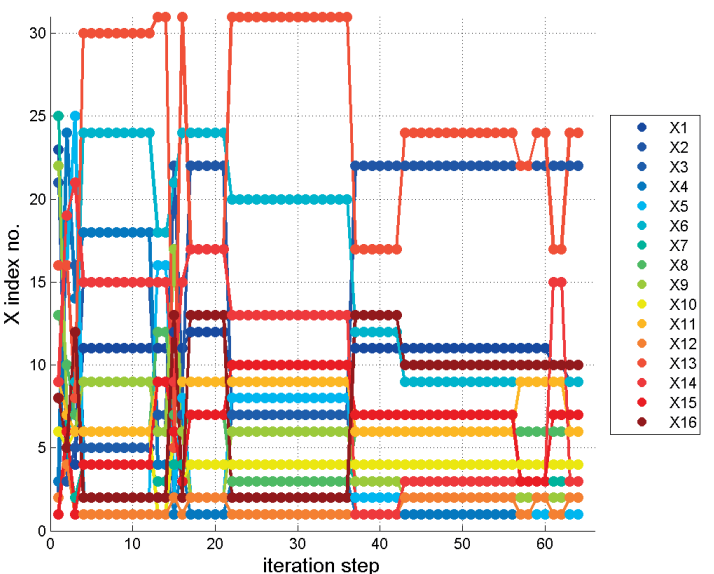

Fig. 12 Initial GA run - History of optimization variables (chosen indexes from vectors of discrete design variables)

\section{Finite element results after optimization using memetic algorithm}

Maximum effective stress after optimization was 120.6 $\mathrm{MPa}$, which was $0.6 \mathrm{MPa}$ above the stress limit. Maximum displacement magnitude was $3.052 \mathrm{~mm}$, which was lower than the displacement limit. The first load factor was 25.93, which was above the minimal buckling load factor. All natural frequencies were outside of the forbidden ranges. All constraints were satisfied except the stress constraint, which was violated only by negligible value. Results can be seen in following Figs. $13-15$ and Tables $2-3$ containing the first 25 natural frequencies.

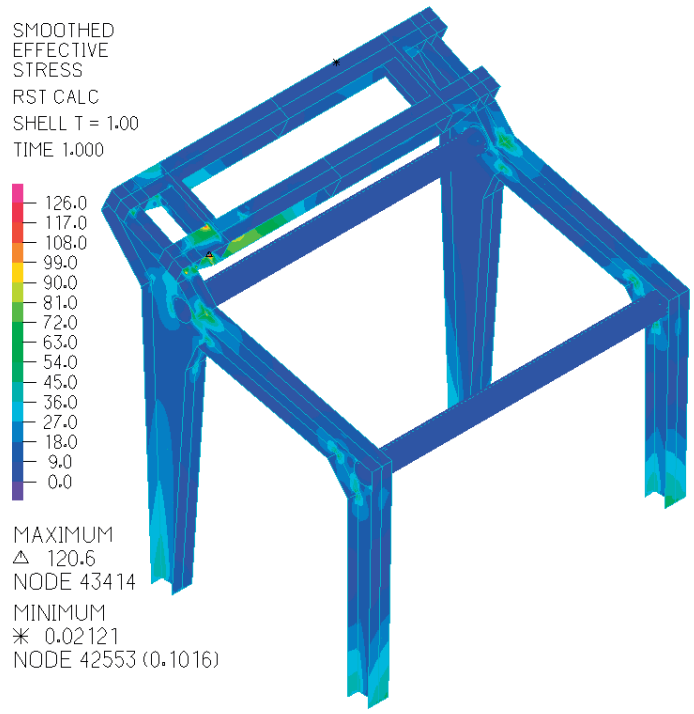

Fig. 13 Smoothed effective stress 


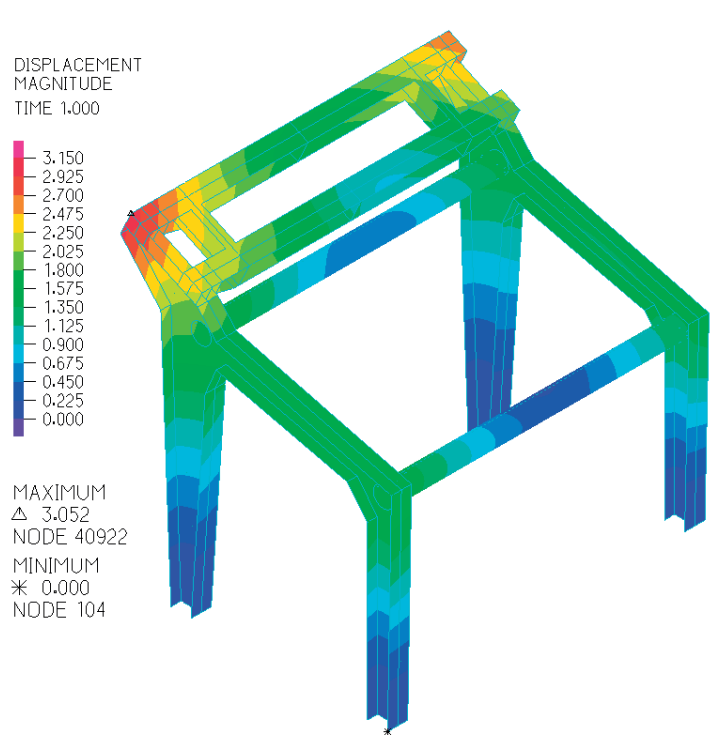

Fig. 14 Displacement magnitude

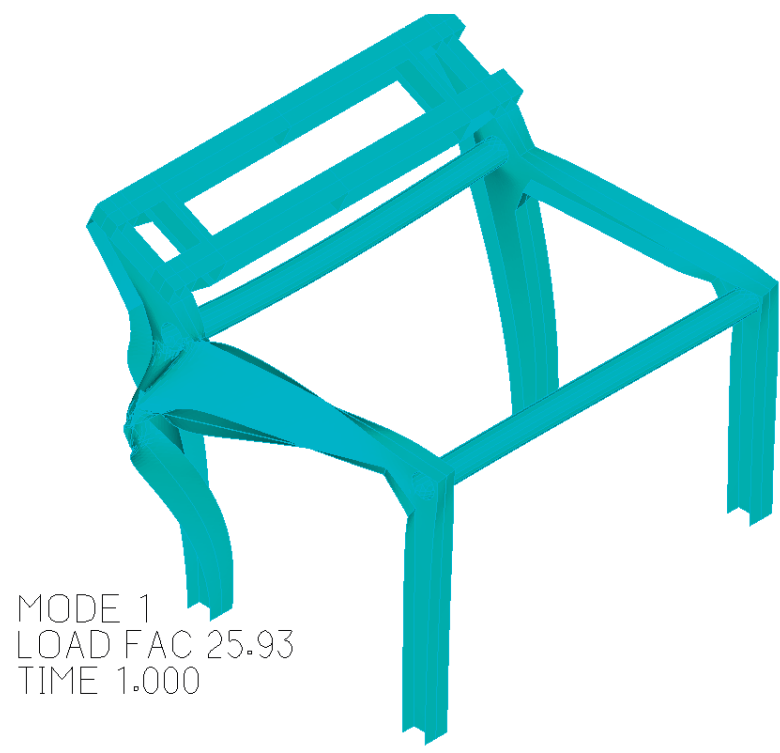

Fig. 15 First buckling mode
Further numerical testing was performed five times for GA and five times for MA to provide statistical data as the tested algorithms used random number generators. The size of GA population was set to 30 . Maximum computational time was set to 8 hours.

Comparison of results for memetic and genetic algorithm

Table 3

\begin{tabular}{|c|c|c|c|c|}
\hline & \multicolumn{2}{|c|}{ MA } & \multicolumn{2}{|c|}{ GA } \\
\hline Run & $f(x)$ & $f(x)$ averaged & $f(x)$ & $f(x)$ averaged \\
\hline 1 & 1467.28 & \multirow{5}{*}{1562.46} & 1573.52 & \multirow{5}{*}{1598.29} \\
\hline 2 & 1448.15 & & 1592.47 & \\
\hline 3 & 1555.42 & & 1534.35 & \\
\hline 4 & 1630.11 & & 1812.25 & \\
\hline 5 & 1711.32 & & 1478.86 & \\
\hline
\end{tabular}

\section{Conclusion}

Optimization of the frame and improvements of design allowed to decrease weight of the frame from the original $2733.5 \mathrm{~kg}$ to $1406.9 \mathrm{~kg}$. The total weight difference is $1326.6 \mathrm{~kg}$ which is roughly $48 \%$ of the original weight. All constraints were satisfied except the stress constraint, which was violated only by $0.5 \%$. Maximum effective stress after optimization was 120.6 MPa, which was 0.6 MPa above the stress limit. Maximum displacement magnitude was $3.052 \mathrm{~mm}$, which was lower than the displacement limit of $5 \mathrm{~mm}$. The first load factor was 25.93 , which was far above the minimal buckling load factor of 6 . All natural frequencies were outside of the forbidden ranges.

Numerical testing showed that the proposed memetic algorithm performs slightly better than classical genetic algorithm. The stress constraint seemed to play major role in penalization of the objective function as it was the most frequent cause of penalization of objective function and the optimized design reached the stress limit. Performance of memetic algorithm could be probably further improved by adding Fully Stress Design method (FSD) to the local search methods, which is

Natural frequencies for 25 first modes after optimization using memetic algorithm

Table 2

\begin{tabular}{|c|c|c|c|c|c|c|c|c|c|}
\hline $\begin{array}{c}\text { Mode } \\
\text { no. }\end{array}$ & $\begin{array}{c}\text { Natural } \\
\text { frequency } \\
{[\mathbf{H z}]}\end{array}$ & $\begin{array}{c}\text { Mode } \\
\text { no. }\end{array}$ & $\begin{array}{c}\text { Natural } \\
\text { frequency } \\
{[\mathrm{Hz}]}\end{array}$ & $\begin{array}{c}\text { Mode } \\
\mathbf{n o .}\end{array}$ & $\begin{array}{c}\text { Natural } \\
\text { frequency } \\
{[\mathrm{Hz}]}\end{array}$ & $\begin{array}{c}\text { Mode } \\
\text { no. }\end{array}$ & $\begin{array}{c}\text { Natural } \\
\text { frequency } \\
{[\mathrm{Hz}]}\end{array}$ & $\begin{array}{c}\text { Mode } \\
\text { no. }\end{array}$ & $\begin{array}{c}\text { Natural } \\
\text { frequency } \\
{[\mathrm{Hz}]}\end{array}$ \\
\hline $\mathbf{1}$ & 0.47 & $\mathbf{6}$ & 4.20 & $\mathbf{1 1}$ & 13.09 & $\mathbf{1 6}$ & 17.51 & $\mathbf{2 1}$ & 26.58 \\
\hline $\mathbf{2}$ & 1.28 & $\mathbf{7}$ & 5.20 & $\mathbf{1 2}$ & 13.24 & $\mathbf{1 7}$ & 18.38 & $\mathbf{2 2}$ & 26.78 \\
\hline $\mathbf{3}$ & 1.96 & $\mathbf{8}$ & 9.10 & $\mathbf{1 3}$ & 13.54 & $\mathbf{1 8}$ & 18.67 & $\mathbf{2 3}$ & 27.12 \\
\hline $\mathbf{4}$ & 2.93 & $\mathbf{9}$ & 10.52 & $\mathbf{1 4}$ & 14.99 & $\mathbf{1 9}$ & 23.38 & $\mathbf{2 4}$ & 31.24 \\
\hline $\mathbf{5}$ & 3.29 & $\mathbf{1 0}$ & 11.42 & $\mathbf{1 5}$ & 17.28 & $\mathbf{2 0}$ & 24.94 & $\mathbf{2 5}$ & 31.69 \\
\hline
\end{tabular}


a specialized optimization method for solving problems involving stress constraint [15 - 16].

The ability of memetic algorithms to be relatively easily tailored for specific problem makes them an interesting tool for a modern designer and allows to provide superior performance when compared to classical global optimization methods like genetic algorithms.

\section{Acknowledgement}

This work has been supported by the Slovak Research and Development Agency under the contract No. APVV-14-0096.

\section{References}

[1] HANDRIK, M., VASKO, M., KOPAS, P., MOZER, V.: The Linear and Nonlinear Stability Loss of Structures Due to Thermal Load. Procedia Engineering, 136, 2016, 359-364.

[2] NOVAK, P., DEKYS, V.: Induction Heating of Inner Rolling Bearing Ring in ANSYS. Manufacturing Technology, 15, 5, 2015, 881885.

[3] VASKO, M., GURAN, A., JAKUBOVICOVA, L., KOPAS, P.: Determination the Contact Stress Depending on the Load Rate of the NU220 Roller Bearing. Communications - Scientific Letters of the University of Zilina, 15, 2, 2013, 88-94.

[4] SAPIETOVA, A., DEKYS, V.: Use of MSC.ADAMS Software Product in Modelling Vibration Sources. Communications - Scientific Letters of the University of Zilina, 18, 1A, 2016, 101-107.

[5] ZMINDAK, M., NOVAK, P., EISNER, I.: Modeling of Strength of Elastic Reinforced Composites by Fiber Inclusions. 17 ${ }^{\text {th }}$ intern. conference on Engineering Mechanics, Svratka: Engineering Mechanics, 2011, 699-702.

[6] SAGA, M., PECHAC, P., JAKUBOVICOVA, L.: Application of Multi-Criteria Optimization to Large-Scale Structures Design. Applied Mechanics and Materials, 693, 2014, 171-176.

[7] MOSCATO, P., COTTA, C.: A Gentle Introduction to Memetic Algorithms. Handbook of Metaheuristics. Springer US, 2003, 105-144.

[8] YAO, X., LIU, Y., LIN, G.: Evolutionary Programming Made Faster. IEEE Transaction on Evolutionary Computation, 3, 2, 1999, 82-102.

[9] HANDRIK, M., SAGA, M., KOPAS, P.: Comparison of Monte-Carlo Method and White Space Searching (in Slovak). Acta Mechanica Slovaca, 2008.

[10] HAGER, W., ZHANG, H.: A Survey of Nonlinear Conjugate Gradient Methods. Pac. J. Optim., 2, 1, 2006.

[11] CERVANTES, A.: Constriction Factors and Parameters, 2005. Retrieved from http://tracer.uc3m.es/tws/pso/parameters.html.

[12] BANSAL, J. C., SINGH, P.K., SARASWAT, M., VERMA, A., JADON, S. S., ABRAHAM, A.: Inertia Weight Strategies in Particle Swarm Optimization. Proc. IEEE Nature and Biologically Inspired Computing (NaBIC), Salamanca, 2011, 19-21.

[13] PECHAC, P., SAGA, M.: Controlling of Local Search Methods' Parameters in Memetic Algorithms Using the Principles of Simulated Annealing. Procedia Engineering, 136, 2016, 70-76.

[14] WEIS, P., MOCILAN, M.: Modal Analysis of the Gearbox Frame. Technolog, 8, 3, 2016, 70-75.

[15] SAGA, M., DUDINSKY, M., PECHAC, P.: Optimization of Thin Shell Structures Using FSD Algorithms. Communications Scientific Letters of the University of Zilina, 14, 3, 2012, 32-38.

[16] PECHAC, P., SAGA, M., GURAN, A., RADZISZEWSKI, L.: Implementation of Memetic Algorithms into Structural Optimization, Communications - Scientific Letters of the University of Zilina, vol. 18, No. 1A, 2016, 64-69. 